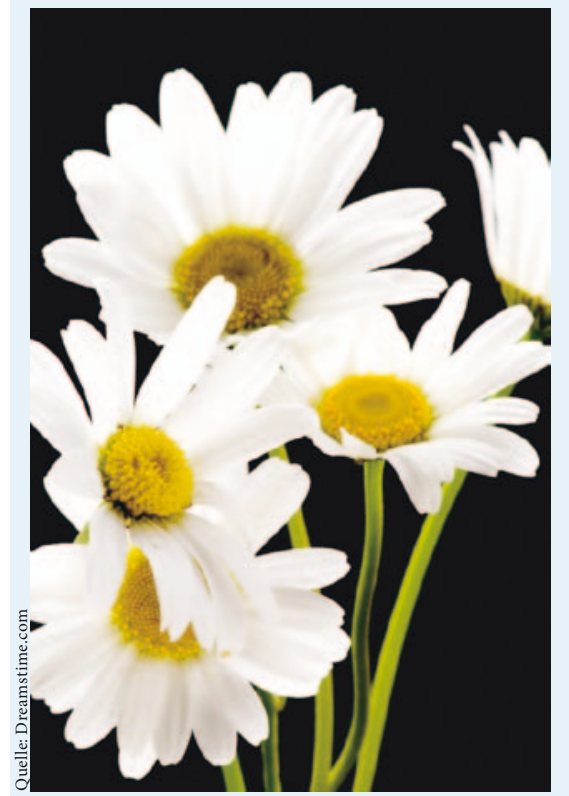

Phytotherapie

Die Kamille ist wohl die bekannteste Heilpflanze hierzulande, seit Langem in Gebrauch, vielfach bewährt und heute immer noch so gut wie früher. Doch auch in der Phytotherapie steht die Zeit nicht still und die Suche nach neuen Wirkstoffen, neuen Indikationen und vielleicht besseren Darreichungsformen geht weiter. Mit welchen Methoden und welchem Aufwand Pharmafirmen, die sich mit pflanzlichen Arzneimitteln beschäftigen, nach Innovationen suchen, steht rechts und auf den nächsten beiden Seiten. Die Suche ist vielleicht etwas einfacher als in der klassischen Pharmaindustrie; die Anforderungen, die die Behörden bei der Zulassung stellen, sind jedoch genauso streng. Einen Biobonus gibt es nicht. Und wie Vertreter der chinesischen, homöopathischen sowie klassischen Phytotherapie ihre Disziplin sehen, lesen Sie im Expertengespräch ab der Seite 203.

\title{
Wenn das Neue das schon lang Bewährte ist
}

Obwohl neue pflanzliche Arzneimittel oft auf den Erkenntnissen der traditionellen Volksmedizin oder Ethnopharmazie beruhen, ist die Phytopharmabranche innovativ. Die Forschungs- und Entwicklungsarbeit bleibt für die Klein- und Mittelunternehmen (KMU) auf dem Feld pflanzlicher Arzneimittel kostspielig und riskant.

Innovation heisst das Zauberwort in der Pharmaindustrie. Die Branche ist ständig auf der Suche nach noch effizienteren und noch innovativeren Wirkstoffen, mit denen die Marktanteile und der künftige Umsatz gesichert werden sollen. Neuentwicklungen sind die Triebfeder eines der wichtigsten Wachstumsträger der Schweizer Wirtschaft. Pro Jahr geben die forschenden klassischen Pharmafirmen hierzulande etwa 6,5 Milliarden Franken für Forschung und Entwicklung aus. Dieser Aufwand zahlt sich medizinisch und vor allem finanziell aus. Ohne eine gefüllte Pipeline sinkt der Aktienkurs und die wirtschaftlichen Aussichten trüben sich ein.

Auch die kleinen und mittleren Pharmaunternehmen, die Phytotherapeutika oder Präparate für die Felder der Komplementärmedizin herstellen, in denen pflanzliche Arzneimittel zum Einsatz kommen, stehen unter einem ähnlichen Innovationsdruck. Wenn es um Präparate geht, die in der Traditionellen Chinesischen Medizin (TCM), der Homöopathie, der anthroposophischen Medizin oder der klassischen Phytotherapie eingesetzt werden, gelten aber auch andere Regeln, wenn es um die Suche nach Innovationen geht. Denn im gleichen Ausmass, wie sie von Branchenriesen der Schulmedizin geleistet werden, sind Forschungsinvestitionen für die KMU kaum bezahlbar. «Wir könnten uns schon rein aus ökonomischen Gründen solche Forschungs- anstrengungen kaum leisten», sagt Jürgen Drewe, Leiter Präklinik der Zeller AG in Romanshorn. Die Mittel, die notwendig sind, um einen vollkommen neuen pflanzlichen Wirkstoff überhaupt zur Marktreife bringen zu können, gehen leicht in den zweistelligen Millionenbereich. $\mathrm{Ob}$ sich diese Investitionen tatsächlich rechnen, ist im Einzelfall kaum zu kalkulieren, und deshalb sind sie ein grosses Wagnis. Die Chance, dass ein Wirkstoff den Weg durch die präklinischen und klinischen Studien bis hin zur Markteinführung schafft, bewegt sich im Bereich zwischen 1:5000 und 1:10 000. Deshalb müssen die grossen Pharmafirmen eine ganze Anzahl von Stoffen in das Rennen um die Zulassung schicken, damit es einige nach dem Ausscheiden der meisten Kandidaten doch noch auf den Markt schaffen. Die müssen dann die Entwicklungskosten für alle Projekte decken und noch einen Gewinn abwerfen. Vielen der KMU, die pflanzliche Arzneimittel herstellen, fehlen dazu die finanziellen Mittel und personellen Ressourcen.

Was die Zulassungsverfahren betrifft, müssen die Phytotherapeutika die gleichen strengen Zulassungsbedingungen erfüllen wie die synthetisch hergestellten Arzneimittel der Schulmedizin. Einen Bonus für die Produkte aus der Natur gibt es nicht, was die Beurteilung der Wirkung, Sicherheit und Wirtschaftlichkeit betrifft.

\section{KARGER}


Viele Wirkstoffe sind längst bekannt Dadurch dass im komplementärmedizinischen Bereich auch für viele bereits bekannte Arzneipflanzen noch offene Forschungsfragen gegeben sind, wie etwa in Hinblick auf neue Anwendungsgebiete, ist hier der Bedarf an völlig neuen Substanzen geringer. Anders als die klassischen Pharmaunternehmen, die dauernd auf der Suche nach noch effizienteren Molekülen für den nächsten Blockbuster sind, kann die Forschung in der Komplementärmedizin auch auf altes medizinisches Wissen und auf über Generationen überlieferte Anwendungen der Volksheilkunde zurückgreifen. In manchen Therapierichtungen profitiert sie auch heute noch von den Ideen der Gründer ihres jeweiligen Bereichs der Komplementärmedizin. «Wir können beim Erarbeiten von Produkten mit innovativen Anwendungsgebieten oder Darreichungsformen immer wieder auf Wirkstoffe zurückgreifen, für die es schon einen langjährigen medizinischen Gebrauch gibt», sagt Günter Meng, Leiter der Forschung und Entwicklung bei der Schwabe GmbH \& Co KG. Deshalb kann die Entwicklungsarbeit an bewährten und deshalb vielleicht auch Erfolg versprechenden Stellen beginnen.

Die traditionelle Medizin bietet tatsächlich einen noch lange nicht ausgeschöpften Fundus von Heilwissen. «Wir orientieren uns bei der Entwicklung unserer Präparate an den Formeln eines alten tibetischen Rezeptbuchs», sagt Herbert Schwabl, VR-Präsident der PADMA AG. Das Unternehmen mit Sitz in Schwerzenbach ist auf Produkte spezialisiert, deren Wirkstoffkombinationen auf Rezepturen der Tibetischen Medizin basieren. Das Wissen dazu stammt etwa aus einem Buch, das dem Firmengründer Karl Lutz um das Jahr 1960 von den Nachfahren eines im 19. Jahrhundert nach Russland ausgewanderten tibetischen Arztes überreicht wurde. «Wir haben erst einen kleinen Teil der dort beschriebenen Präparate zu westlich anerkannten Arzneimitteln gemacht», sagt er. In den nächsten Jahren und wahrscheinlich auch noch Jahrzehnten kann sich die PADMA AG bei der «Entwicklung» neuer Präparate aus diesem tradierten Wissen alimentieren. Deshalb ist es kaum notwendig, neue Rezepturen für neue Indikationen zu entwickeln. Innovationen lassen sich deshalb eher durch Anpassung der traditionellen Formeln an moderne Qualitätsanforderungen, wie z.B. die botanische Definition der verwendeten Arzneipflanzen oder die Verbesserungen in der Darreichungsform durch den Einsatz von Kapseln, erzielen. «Ein Teil der Entwicklungsarbeit geht deshalb auch in diese Richtung, ein anderer Teil in die Entwicklung eines tibetischen Präparats, welches bei Verdauungsschwäche angewendet wird», sagt er. Ähnlich zeigt sich das Bild bei Pflanzenpräparaten, deren Wirkung aus der TCM überliefert ist. Auch hier hält die Tradition noch genügend Wissen bereit, das durch Präparate noch gar nicht in die medizinische Praxis im Westen umgesetzt worden ist.

\section{Rudolf Steiners Anregungen \\ sind ein reicher Fundus}

Auch in anderen Bereichen der Komplementärmedizin ist das Wissen umfangreich, das noch ungenutzt in der Literatur dokumentiert ist. So etwa in der anthroposophischen Medizin. «Rudolf Steiner hat eine grosse Zahl von Anregungen hinterlassen, welche Pflanzen bei welcher Indikation und in welcher Zubereitungsart wirken könnten», sagt Martin Schnelle, Chef von Forschung und Entwicklung der Weleda AG in Arlesheim. «Wir verfügen über einen grossen Ideenschatz, der zum Teil noch aus der Gründerzeit der Firma, zum Teil von Rudolf Steiner selbst stammt.» Anthroposophisch arbeitende Ärzte und die Weleda entdecken immer wieder interessante und unbekannte Zusammenhänge zwischen Heilpflanzen und dem Behandlungserfolg und entwickeln daraus auch neue Medikamente. DerWissenstransfer von der Praxis in das Unter-

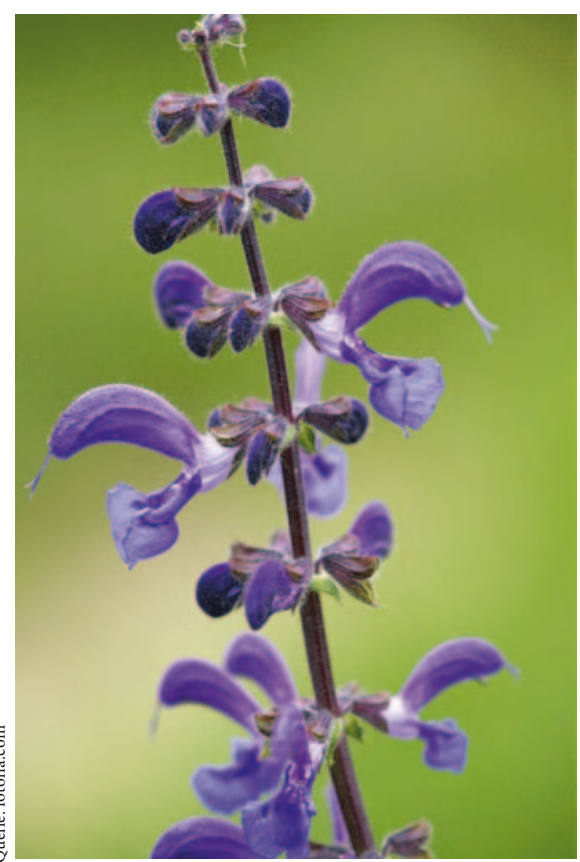

Abb. 1. Salvia pratensis (Wiesensalbei).

nehmen soll bei Weleda noch intensiviert werden; regelmässige Treffen mit anthroposophisch arbeitenden Medizinern und Kliniken gehören zum Alltag der Forscher. Bei einem Projekt hat sich Weleda allerdings von anderen Ideen beeinflussen lassen als von den Steinerschen. In den letzten Jahren haben die Forscher auch an einem Cannabis-Präparat gearbeitet, für das sie im Herbst bei der Swissmedic die Zulassung beantragen wollen.

Eine wichtige Quelle für neue Ideen zur Wirksamkeit von Pflanzen ist bei Zeller die Ethnopharmazie. Während in Mitteleuropa das Einsatzspektrum der meisten Heilpflanzen gut bekannt und seit den Zeiten Hildegards von Bingen auch gut dokumentiert ist, ist dasjenige in aussereuropäischen $\mathrm{Ge}$ bieten noch weitgehend unerforscht. «Wir beziehen viele Pflanzen aus solchen Gebieten und versuchen vom Wissen vor Ort zu lernen», sagt Drewe. Lokale Partner können in manchen Fällen Hinweise geben, wozu einzelne Pflanzenarten dort eingesetzt werden.

Auch für die Firma Schwabe stellt die Arbeit mit Pflanzen aus fernen Ländern eine grosse Herausforderung dar. Ein Grund liegt in der kulturellen Schwierigkeit, die mit solchen Projek- 


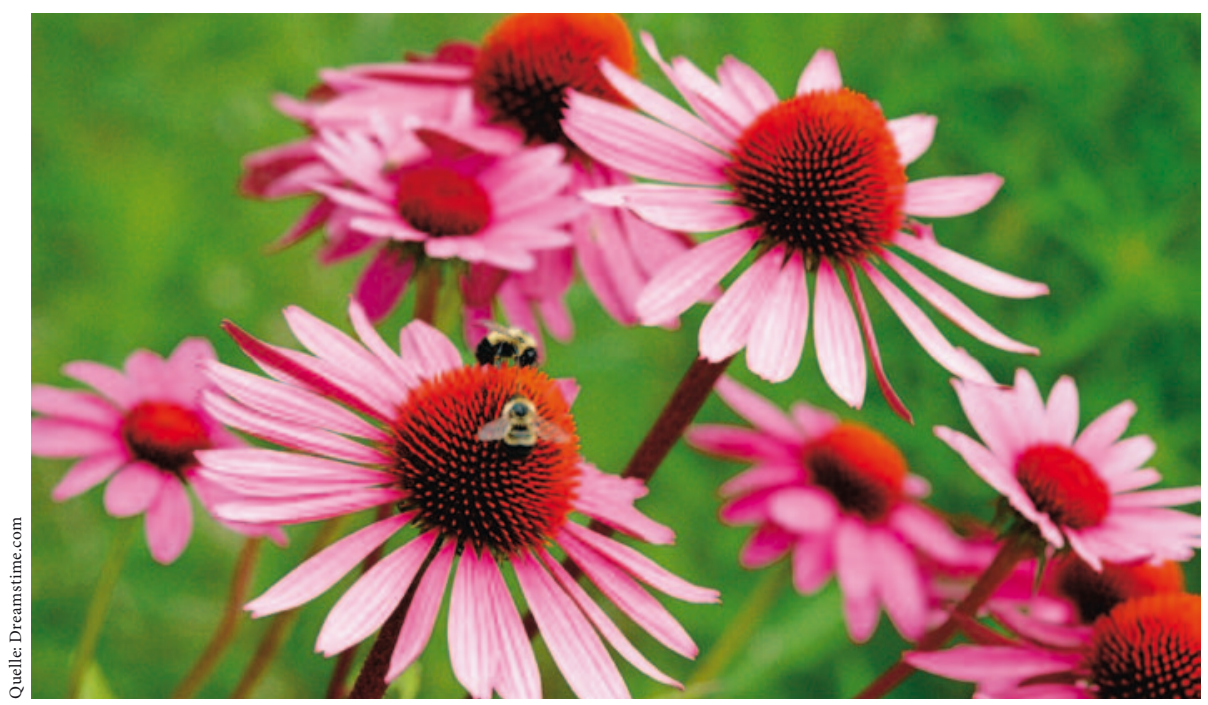

Abb. 2. Echinacea purpurea (Purpurfarbener Sonnenhut).

ten verbunden ist. "Es ist nicht einfach, den Menschen, mit denen man vor Ort zusammenarbeitet, nahezubringen, was wir machen und brauchen», sagt Schwabe-Entwicklungschef Günter Meng. In bestimmten Kulturen, in denen das Wissen nur mündlich und wenig systematisch weitergegeben werde, sei es für die westlichen Mediziner auch häufig schwierig, sich ein Bild von der Pflanze und ihrer Wirkung zu machen. Anders hingegen liege der Fall in Kulturen, die über eine lange medizinische Tradition verfügen und deren Heilwissen systematisch organisiert ist. "Aus der Chinesischen Medizin lässt sich deshalb eine ganze Menge für die Entwicklung von pflanzlichen Arzneimitteln lernen», sagt er. Auch die Heilkundeliteratur Europas ist für die Forscher des Unternehmens ein wichtiger Fundus.

Neben diesem Ansatz verfolgen die Wissenschafter von Schwabe auch den klassischen Ansatz der Pharmazie bei der Suche eines geeigneten Wirkstoffs zur Behandlung einer bestimmten Erkrankung. Aus den Pflanzen wird ein Inhaltsstoffmuster isoliert und in einem Screening die Wirkung getestet. "Mit diesem Verfahren haben wir mehrere Projekte sehr weit vorantreiben können", sagt Meng. Eines davon ist ein in Deutschland kürzlich zugelassener Pflanzenextrakt, der Eigenschaften zur Behandlung von
Symptomen bei Ängstlichkeit hat. Schwabe hat mit etwa 125 Mitarbeitern in Forschung und Entwicklung sowie einem entsprechenden Budget im zweistelligen Millionenbereich auch die finanzielle Kraft, um neue Wirkstoffe systematisch zu testen.

Auch Zeller beschreitet den klassisch pharmazeutischen Weg bei der Entwicklung neuer Phytopräparate. Als Ergebnis von Bio-Assay-unterstützten Screening-Untersuchungen versprechen sich die Forscher des Unternehmens neue Einsatzmöglichkeiten verschiedener pflanzlicher Extrakte. So haben sich die Forscher des Unternehmens mithilfe von Bio-Assays auf die Suche nach pflanzlichen Wirkstoffen gemacht, die beim metabolischen Syndrom, einer Störung im Glukose- oder Fettstoffwechsel, helfen könnten. «Wir haben verschiedene molekulare Angriffspunkte untersucht, mit denen Wirkstoffe aus Pflanzen interagieren können», sagt Forschungschef Jürgen Drewe. Wenn Phytotherapeutika das gesamte Verfahren aus klinischen Studien und $\mathrm{Zu}$ lassungsverfahren durchlaufen müssen, ist leicht mit einem Zeitaufwand zwischen fünf und zehn Jahren zu rechnen. Bei KMU, bei denen die personellen und finanziellen Möglichkeiten beschränkt sind, sprengen Forschung und Entwicklung rasch die Grenzen der Kapazität. Wie in vielen anderen Branchen auch, wer- den deshalb solche Aufgaben ausgelagert, weil die Ressourcen «in-house» es nicht erlauben, alles selbst $\mathrm{zu}$ machen.

Forschungskooperationen sind wichtig Viele Unternehmen finden über Forschungskooperationen einen Weg, um zu neuen Erkenntnissen zu kommen. Der Zugang zu neuem Wissen ist auch für die kleinen und mittleren im Phytotherapeutikasektor tätigen Pharmaunternehmen wichtig, die sich eine systematische Entwicklungsarbeit in grösserem Stil kaum leisten können. Sie profitieren deshalb von der Möglichkeit, in internationalen Forschungsprogrammen mit Hochschulen und auch anderen Unternehmen kooperieren zu können. Diesen Vorteil können sich auch Schweizer Firmen zunutze machen: Durch eine Vereinbarung zwischen Bern und Brüssel können sie sich an den Europäischen Rahmenforschungsprogrammen beteiligen, die bereits seit mehr als 25 Jahren laufen und die sich als Keilriemen des Wissenstransfers von der Wissenschaft in die Wirtschaft bewährt haben. An den Projekten des Rahmenprogramms arbeiten grenzübergreifend Unternehmen und Hochschulen zusammen. Wissenschafter von Universitäten im In- und Ausland lösen gemeinsam mit den Spezialisten der beteiligten Firmen Probleme. Es geht konkret um die Entwicklung von Produkten, die sich auf dem internationalen Markt für Arzneimittel behaupten können. Dabei wird nicht Grundlagenforschung betrieben, sondern angewandte Wissenschaft, die früher oder später einmal zu Produkten führen soll, die Patienten helfen. Ohne diese EU-Förderung wären auch viele der Schweizer Phytotherapie-KMU gar nicht in der Lage, diese Entwicklungsarbeit zu leisten. Finanziell und von der eigenen Personalsituation her kämen sie rasch an die Grenze des Zumutbaren. Und viele Kenntnisse und Anregungen, die noch in der Literatur versteckt sind, müssten weiter der Umsetzung in Produkte harren. (rfi) 